\title{
EFFECTS OF VESTIBULAR LOSS ON HEAD STABILIZATION IN RESPONSE TO HEAD AND BODY PERTURBATIONS
}

\author{
J Vestibular Res 1996;6:423-37 \\ C.L. Shupert and F.B. Horak
}

We wish to correct two errors in the above publication.

The first error occurs on page 433 in the second full paragraph of the right-hand column. The sentence that reads "Allum and colleagues have reported that vestibular loss patients responding to platform perturbations show abnormally low activation amplitudes in paraspinal muscles (22)" should read ". . . show abnormally high activation amplitudes in paraspinal muscles (22)."

Second, for reference 22, two co-authors were inadvertently omitted. The authors should read "Allum JHJ, Honegger F, and Schicks H."

We regret any inconvenience these errors may have caused. 